\title{
Management of the urethra in urothelial bladder cancer
}

\author{
Androniki Kanaroglou, MD; Bobby Shayegan, MD, FRCSC ${ }^{\dagger}$
}

\begin{abstract}
The standard of care in the management of invasive urothelial cancer of the bladder is radical cystectomy and pelvic lymphadenectomy. Although uncommon, recurrence of disease in the retained urethra following cystectomy carries a poor prognosis. The need for assessment of risk of recurrence is greater now than ever with wider adoption of orthotopic bladder substitution. This review will address the contemporary management of the urethra following cystectomy for urothelial cancer.
\end{abstract}

Can Urol Assoc J 2009;3(Suppl4):S211-4

\section{Introduction}

Radical cystectomy with pelvic lymphadenectomy remains the benchmark in the treatment of invasive urothelial bladder cancer. With increasing enthusiasm for orthotopic urinary diversion and the added morbidity associated with urethrectomy, there has been a paradigm shift in the management of the urethra over the last few decades. Currently, urethrectomy in conjunction with cystectomy is rarely performed due to evidence indicating an overall low incidence of urethral recurrence (UR) following cystectomy. Moreover, studies conducted in the 1970s demonstrated no survival advantage with prophylactic urethrectomy. ${ }^{1,2}$ Nonetheless, in selected patients, urethrectomy remains appropriate at time of cystectomy. This review addresses the management of the urethra in patients who have undergone cystectomy for urothelial carcinoma of the bladder.

\section{Incidence}

Urethral involvement in urothelial cancer may be synchronous or metachronous. Synchronous disease is present at the time of cystectomy but goes unrecognized; metachronous disease may arise from tumour spillage and implantation, field change (de novo) or contiguous invasion from a positive apical margin. Studies assessing synchronous disease suggest that when en bloc prophylactic urethrectomies are performed, $57 \%$ to $100 \%$ of the time no ure- thral tumour is found. ${ }^{1}$ Several other studies have confirmed similarly low rates of urethral involvement in both male and female cystectomy specimens. ${ }^{3-6}$

The overall incidence of UR following cystectomy is approximately $8 \%$ to $10 \%{ }^{1}$ In men, incidence ranges from $4 \%$ to $7.9 \%{ }^{7-10}$ Women seem to have at least comparable UR rates in contemporary series, ranging from $4.7 \%$ to $12 \% .5,11,12$ Interestingly, recurrence rates have also been shown to vary by type of diversion performed. In patients with cutaneous urinary diversions, recurrence rates range from $2.1 \%$ to $11.1 \%$, while in those with orthotopic bladder substitutions, recurrence rates are slightly lower at $0.5 \%$ to $4.3 \%$. $^{7-11,13,14}$

Median time to recurrence ranges from 12.8 to 56 months across studies, but most patients recur within 3 years. ${ }^{7-11}$ There are reports of delayed recurrences at 10 and 20 years, thus highlighting the importance of proper lifelong monitoring. ${ }^{1}$ In one study comparing ileal conduits to neobladders, average recurrence time was 2 years and no significant difference was found between the 2 groups. ${ }^{9}$ Based on one study where all female recurrences occurred more than 3 years after cystectomy, it appears that recurrence may occur later in women. ${ }^{11}$ This has been postulated to be due to the shorter length of remnant urothelium, or due to the process of aging resulting in cranial migration of metaplastic squamous cell mucosa in the urethra, which may have a protective effect. ${ }^{5}$

\section{Risk factors for recurrence}

Several pathologic variables have been noted either to be associated with synchronous disease in the urethra, or to predict a higher probability of metachronous recurrence in the retained urethra. Unfortunately, there is conflicting evidence across series as to the individual significance of these variables. Nonetheless, with increasing numbers of orthotopic bladder substitutions performed for urothelial bladder cancer, a review of the current evidence is appropriate.

\section{Male risk factors}

In a large retrospective review of 729 male cystectomy cases, multivariate analysis showed that independent predictors 
of UR included previous history of non-muscle-invasive bladder cancer (which may suggest multifocality), pathologic subgroup (pTis, pTa, pT1) and prostatic involvement. ${ }^{10}$ In that analysis, the latter was the most significant risk factor, which remains the only consistent finding across all series. ${ }^{1,7-9,13}$ In 1996, researchers at the University of Southern California (USC) published a retrospective review of 436 men undergoing cystectomy and found that the 5-year probability of UR was only $6 \%$ if there was no prostatic involvement, but increased to $15 \%$ and $21 \%$ with superficial and stromal invasion of the prostate, respectively. ${ }^{8}$ Another retrospective review of 768 male cystectomies from the same centre confirmed similar findings, showing that with no prostatic involvement, 5 -year risk of UR is $5 \%$, rising to $12 \%$ and $18 \%$ with superficial and stromal invasion, respectively. They also found that carcinoma in situ (CIS) and multifocality were not statistically significant for increased UR risk. ${ }^{9}$ In a third retrospective paper from Vanderbilt University, 196 radical cystectomy patients with orthotopic neobladders were reviewed. Nearly half of the final pathology specimens contained CIS; however, only 1 patient developed UR at 5.5 years postoperatively. ${ }^{13}$ Others have also suggested that while neither CIS nor multifocality independently increases UR risk, they independently raise the risk of prostatic involvement, which in turn raises the risk of UR. ${ }^{7,8,15}$ Collectively, the current evidence supports prostatic stromal invasion as the single most significant risk factor for UR.

Given the importance of prostatic stromal invasion, preoperative prostatic biopsies have been proposed as a necessary step in determining patient eligibility for orthotopic bladder substitution. However, a prospective series by Lebret and colleagues demonstrated that intraoperative frozen section of the apical margin was more accurate than preoperative assessment. ${ }^{16}$ Most recently, Kassouf and colleagues confirmed that preoperative prostatic urethral biopsies have limited usefulness in predicting final urethral margin status. ${ }^{17}$ It is now routine in most centres performing orthotopic neobladders to perform intraoperative frozen section of the apical prostatic margin. Patients must be made aware that presence of disease at frozen section will necessitate cutaneous diversion (either continent or incontinent) and possibly urethrectomy (either simultaneously or in a staged procedure).

\section{Female risk factors}

The fate of retained female urethra is a relatively new concept given that urethrectomy was routinely performed en bloc with the bladder and anterior vaginal wall. Unlike the situation in male patients, urethrectomy in females adds little if any morbidity to cystectomy. However, in recent years, orthotopic bladder substitution in women has become more widely accepted. Accordingly, several studies have examined the risk of UR in female patients with urothelial bladder cancer.

In 1995, Stein and colleagues reviewed archival specimens from 67 women undergoing radical cystectomy and found a highly significant association between tumour involvement at the bladder neck and the urethra $(p<0.0001)$. In this study, patients with urethral cancer also had bladder neck involvement, and all patients with a tumour-free bladder neck had no evidence of urethral tumour. ${ }^{18}$ In a recent univariate analysis, Akkad and colleagues found that multifocality and tumour at the bladder neck predicted urethral recurrence, whereas CIS and nodal status did not. ${ }^{11}$ In a retrospective review of 356 females in the same year, Stenzl and colleagues reported that $2 \%$ of specimens had urethral involvement at initial presentation, and that tumour at the bladder neck $(p<0.0001)$ and trigone $(p<0.035)$ were most often the regions of primary tumour in these patients. They concluded that the only consistent risk factor is bladder neck involvement. ${ }^{19}$ Indeed, the most widely accepted risk factor in the literature for urethral involvement in women is tumour at the bladder neck. However, it should be pointed out that while gross tumour in the urethra mandates an en bloc urethrectomy, there is no consensus on the management of the urethra when the bladder neck is involved.

\section{Impact of urinary diversion type}

Some retrospective studies have noted higher recurrence rates in cutaneous diversions when compared to orthotopic substitutions. The original study by Freeman and colleagues determined that patients with a Kock ileal neobladder were at significantly lower risk for UR compared with those who underwent a nonorthotopic diversion, with UR rates of only $2.9 \%$ in the former group compared to $11.1 \%$ in the latter (mean recurrence time of 2.3 and $1.6 \mathrm{yr}$, respectively). Even in the presence of prostatic involvement, the neobladder group had lower recurrence rates compared with the conduit group. ${ }^{8}$ On multivariate analysis, Stein and colleagues found that risk of UR in orthotopic diversions was $4 \%$ and increased to $9 \%$ with cutaneous diversion. If there was no prostatic involvement, the UR rate with orthotopic diversion was only $3 \%$ at 5 years compared with $8 \%$ among those who had cutaneous diversions. In patients with prostatic stromal involvement and an orthotopic diversion UR was $11 \%$, but increased to $24 \%$ in those with a cutaneous diversion. ${ }^{9}$

To explain these findings, some have suggested that the juxtaposed ileum intrinsically resists malignancy or passively confers resistance to UR. Continued urine flow may also have protective effects on the remnant urothelium. ${ }^{8,13}$ Others feel that selection bias sufficiently accounts for the different UR rates, where cutaneously diverted patients are older and have higher rates of prostatic and nodal involvement. ${ }^{7,10}$ 


\section{Postoperative monitoring of the urethra}

If a urethral remnant is left in situ, the patient should be monitored for recurrence for life. Surveillance options include 1) routine cytology, either voided or by urethral washing; 2) routine urethroscopy; or 3) performing cytology and/or urethroscopy only when indicated by history or physical (e.g., hematuria, bloody discharge, pain, palpable mass, change in voiding habits). There is no consensus on optimal monitoring technique or timing regimen, but many authors advocate at least biannual exams for the first 3 years, with decreasing frequency thereafter. $1,7,9,11,14$

Some studies included comparisons of surveillance techniques. Data from USC suggested that symptomatic presentation occurred later, with a median recurrence time of 30.9 months, versus 11.6 months for those presenting with positive cytology. ${ }^{14} \mathrm{~A}$ European review found that cytology effectively detected superficial recurrences, while symptoms usually heralded invasive recurrences. ${ }^{10}$ Investigators at Memorial Sloan-Kettering Cancer Center questioned whether screening method actually predicts overall survival. ${ }^{20}$ They found that monitoring urethral cytology did not increase survival in patients with recurrence when compared to patients who presented with symptoms. Despite this finding, they continue to advocate cytologic surveillance since it is inexpensive, minimally invasive and relatively easy to perform. Overall, data remains sparse and inconclusive, and so the optimal postoperative monitoring method is a source of ongoing debate.

\section{Management of recurrence and survival}

Experts agree that recurrence should be treated with complete urethrectomy, meatectomy, and conversion to a cutaneous conduit if orthotopic substitution was originally performed. 1,21,22 Despite appropriate management, urethral recurrence postcystectomy carries a relatively poor median overall and disease-specific survival. ${ }^{1,23,24}$ At USC, the median survival with UR was found to be to be 28 months. ${ }^{14}$ The more recently published European data on 729 male patients found the median survival post-UR was 53.8 months with a 5 -year actuarial survival of $43 \% .{ }^{10}$ In this group, superficial urethral disease did not carry a better prognosis than invasive urethral disease; however, reports on the relative impact of urethral and bladder pathology on diseasespecific survival are contradictory. ${ }^{1,14,19,25}$

\section{Conclusion}

Urethral recurrence post-radical cystectomy is a relatively rare phenomenon, occurring in $8 \%$ to $10 \%$ of patients. In men, the most significant risk factor is prostatic involvement, while in women it is bladder neck involvement. Recent evidence also suggests that orthotopic substitutions may actually have a protective effect on the remnant urethra. Postoperatively, lifelong monitoring of the urethral remnant is necessary. It is not clear whether routine postoperative cytology has a survival impact, though it is generally advocated. Survival with UR is relatively poor, and all recurrences should be treated with complete urethrectomy and meatectomy.

In the author's experience, urethral recurrence following cystectomy also occurs quite uncommonly. Most patients who relapse do so with systemic disease and there is rarely a need for delayed urethrectomy. In those deemed to be at high risk of urethral relapse (such as patients with positive apical frozen sections), total urethrectomy at the time of cystectomy may be entertained. However, we do not routinely perform urethrectomy in patients with prostatic involvement if frozen sections of the apical urethral margin are negative.

*Senior resident, Division of Urology; ${ }^{\dagger}$ Assistant Professor, Urologic Oncology, Department of Surgery, McMaster University, Hamilton, ON

Competing interests: None declared.

This paper has been peer-reviewed.

\section{References}

1. Clark PE, Hall MC. Contemporary management of the urethra in patients after radical cystectomy for bladder cancer. Urol Clin North Am 2005;32:199-206.

2. Schellhammer PF, Whitemore WF Jr. Transitional cell carcinoma of the urethra in men having cystectomy for bladder cancer. J Urol 1976;115:56-60.

3. Tobisu K, Tanaka Y, Mizutani T, et al. Transitional cell carcinoma of the urethra in men following cystectomy for bladder cancer: multivariate analysis for risk factors. J Urol 1991;146:1551-3; discussion 1553-4.

4. Tobisu $K$, Kanai $Y$, Sakamoto $M$, et al. Involvement of the anterior urethra in male patients with transitional cell-carcinoma of the bladder undergoing radical cystectomy with simultaneous urethrectomy. Jpn I Clin Oncol 1997;27:406-9.

5. Stein JP, Penson DF, Wu SD, et al. Pathological guidelines for orthotopic urinary diversion in women with bladder cancer: a review of the literature. J Urol 2007;178(3P+1):756-60.

6. Stein JP, Esrig D, Freeman JA, et al. Prospective pathologic analysis of female cystectomy specimens: risk factors for orthotopic diversion in women. Urology 1998;51:951-5.

7. Nieder AM, Sved PD, Gomez P, et al. Urethral recurrence after cystoprostatectomy: implications for urinary diversion and monitoring. Urology 2004;64:950-4.

8. Freeman JA, Tarter TA, Esrig D, et al. Urethral recurrence in patients with orthotopic ileal neobladders. $J$ Urol 1996;156:1615-9.

9. Stein JP, Clark P, Miranda $G$, et al. Urethral tumor recurrence following cystectomy and urinary diversion: clinical and pathological characteristics in 768 male patients. J Urol 2005; 173:1163-8.

10. Huguet J, Monllau V, Sabaté S, et al. Diagnosis, risk factors, and outcome of urethral recurrences following radical cystectomy for bladder cancer in 729 male patients. Eur Urol 2008;53:785-92; discussion 792-3.

11. Akkad T, Gozzi C, Deibl M, et al. Tumor recurrence in the remnant urothelium of females undergoing radical cystectomy for transitional cell carcinoma of the bladder: long-term results from a single center. J Urol 2006;175:1268-71; discussion 1271.

12. Ali-el-Dein B, Abdel-Latif $M$, Ashamallah A, et al. Local urethral recurrence after radical cystectomy and orthotopic bladder substitution in women: a prospective study. J Urol 2004;171:275-8. 
13. Hassan JM, Cookson MS, Smith JA Jr, et al. Urethral recurrence in patients following orthotopic urinary diversion. J Urol 2004;172(4Pt1):1338-41.

14. Clark PE, Stein JP, Groshen SG, et al. The management of urethral transitional cell carcinoma after radical cystectomy for invasive bladder cancer. J Urol 2004;172(4P+1):1342-7.

15. Nixon RG, Chang SS, Lafleur BJ, et al. Carcinoma in situ and tumor multifocality predict the risk of prostatic urethral involvement at radical cystectomy in men with transitional cell carcinoma of the bladder. J Urol 2002;167(2P+1):502-5.

16. Lebret T, Hervé JM, Barré P, et al. Urethral recurrence of transitional cell carcinoma of the bladder. Predictive value of preoperative latero-montanal biopsies and urethral frozen sections during prostatocystectomy. Eur Urol 1998;33:170-4.

17. Kassouf W, Spiess PE, Brown GA, et al. Prostatic urethral biopsy has limited usefulness in counseling patients regarding final urethral margin status during orthotopic neobladder reconstruction. J Urol 2008; 180:164-7; discussion 167.

18. Stein JP, Cote RJ, Freeman JA, et al. Indications for lower urinary tract reconstruction in women after cystectomy for bladder cancer: a pathological review of female cystectomy specimens. J Urol 1995; 154:1329-33.

19. Stenzl A, Draxl H, Posch B, et al. The risk of urethral tumors in female bladder cancer: can the urethra be used for orthotopic reconstruction of the lower urinary tract? J Urol 1995;153(3P+2):950-5.
20. Lin DW, Herr HW, Dalbagni G. Value of urethral wash cytology in the retained male urethra after radical cystoprostatectomy. J Urol 2003;169:961-3.

21. Nelles IL, Konety BR, Saigal C, et al. Urologic Diseases in America Project. Urethrectomy following cystectomy for bladder cancer in men: practice patterns and impact on survival. J Urol 2008; 180:1933-6; discussion 1936-7.

22. Freeman JA, Esrig D, Stein JP, et al. Management of the patient with bladder cancer. Urethral recurrence. Urol Clin North Am 1994;21:645-51.

23. Poole-Wilson DS, Barnard RJ. Total cystectomy for bladder tumours. Br J Urol 1971;43:16-24.

24. Zabbo A, Montie JE. Management of the urethra in men undergoing radical cystectomy for bladder cancer. J Urol 1984;131:267-8.

25. Schellhammer PF, Whitemore WF Jr. Transitional cell carcinoma of the urethra in men having cystectomy for bladder cancer. J Urol 1976;115:56-60.

Correspondence: Dr. Bobby Shayegan, McMaster Institute of Urology, St. Joseph's Healthcare, 50 Charlton Avenue East, Hamilton, ON L8N 4A6; fax: 905-308-7210; shayeb@mcmaster.ca 